\title{
Stabilization of Oscillations through Backstepping in High-dimensional Systems
}

\author{
Javier Aracil ${ }^{1}$, Francisco Gordillo ${ }^{1}$ and Enrique Ponce ${ }^{2}$ \\ ${ }^{1}$ Dep. de Ingeniería de Sistemas y Automática \\ ${ }^{2}$ Dep. de Matemática Aplicada II, \\ Escuela Superior de Ingenieros, Universidad de Sevilla \\ Camino de los Descubrimientos s/n, \\ 41092-Sevilla (Spain) \\ E-mail:aracil@esi.us.es
}

\begin{abstract}
This paper introduces a method for obtaining stable and robust self-sustained oscillations in a class of single input nonlinear systems of dimension $n \geq 2$. The oscillations are associated to a limit cycle that is produced in a second-order subsystem by means of an appropriate feedback law. Then, the controller is extended to the full system by a backstepping procedure. It is shown that the closed-loop system turns out to be generalized Hamiltonian and that the limit cycle can be thought as born in a Hopf bifurcation after moving a parameter.
\end{abstract}

Keywords: Non-linear Oscillations, Limit Cycle Stabilization, Backstepping Control, Generalized Hamiltonian Systems, Hopf Bifurcation.

\section{INTRODUCTION AND STATEMENT OF THE PROBLEM}

Self-sustained oscillations are one of the distinctive behavioral characteristics of nonlinear systems. Whenever an oscillatory behavior is found or is to be built, there is or must be introduced an underlying nonlinearity. In this paper, a procedure to obtain a nonlinear feedback law that renders a class of single input cascade systems oscillatory is introduced. The oscillation is associated with a stable limit cycle and therefore it is self-sustained and robust. The method is based on matching the open-loop system to a closed-loop one that displays such a stable limit cycle. The feedback law is obtained in two steps. In the first step, a second-order subsystem is controlled to yield a robust nonlinear oscillator. To this end, a fourth degree polynomial Lyapunov function is introduced that guarantees the appropriate properties. Then, the cascade structure of the open-loop system allows us to apply backstepping to recursively obtain the feedback law for the full system. A very appealing byproduct is that the closed-loop system obtained has a generalized Hamiltonian structure [1].

The problem considered here is, therefore, the synthesis of limit cycles and belongs to the class of so-called inverse problems in dynamical systems. Several authors have considered this problem in the past (see for instance [2], [3], [4] and references therein) by working with systems of moderate dimension. One of the interests of the algorithm proposed in this paper is its ability to cope with arbitrary dimensions. Related material can be found in [5].

To set the problem under study in a precise form, consider the cascade systems for which the backstepping method is applicable. In particular, we will be concerned with the special class of strict-feedback systems [6] given by:

$$
\begin{aligned}
\dot{x}_{1} & =x_{2} \\
\dot{x}_{2} & =f_{0}\left(x_{1}, x_{2}\right)+g_{0}\left(x_{1}, x_{2}\right) x_{3} \\
\dot{x}_{3} & =f_{1}\left(x_{1}, x_{2}, x_{3}\right)+g_{1}\left(x_{1}, x_{2}, x_{3}\right) x_{4} \\
& \vdots \\
\dot{x}_{n-1} & =f_{m-1}\left(x_{1}, x_{2}, \ldots, x_{n-1}\right)+g_{m-1}\left(x_{1}, x_{2}, \ldots, x_{n-1}\right) x_{n} \\
\dot{x}_{n} & =f_{m}\left(x_{1}, x_{2}, \ldots, x_{n}\right)+g_{m}\left(x_{1}, x_{2}, \ldots, x_{n}\right) u
\end{aligned}
$$

with $m=n-2$ and $g_{i} \neq 0, \forall i$ in the domain of interest. The form for the first equation is quite usual, mainly in mechanical and electrical systems.

Our goal is to design a feedback law $u$ for system (1) that causes it to oscillate in a stable and robust way. This will be obtained through a controller such that the closed-loop system displays a limit cycle as a limit set. This limit cycle is responsible for the oscillatory behavior.

The paper is organized as follows. In Section II, for $n=2$, a control law that renders systems of the form (1) oscillatory is proposed. Next, in Section III the law is extended to arbitrary dimension $n$. The paper closes with a section of conclusions and some technical details are relegated to the Appendix.

Published in IEEE Transactions on Automatic Control, Vol. 50, No. 5, May 2005, pp. 705-710. DOI: 10.1109/TAC.2005.847038. 


\section{OSCILLATIONS IN TWO-DIMENSIONAL SYSTEMS}

We start with the subsystem formed by the first two equations of (1)

$$
\begin{aligned}
& \dot{x}_{1}=x_{2}, \\
& \dot{x}_{2}=f_{0}\left(x_{1}, x_{2}\right)+g_{0}\left(x_{1}, x_{2}\right) x_{3},
\end{aligned}
$$

where $x_{3}$ has to be interpreted as a virtual control $x_{3}=\alpha_{0}\left(x_{1}, x_{2}\right)$. Now we design a feedback law to render this twodimensional subsystem oscillatory. To that end we adopt as reference behavior that of the nonlinear oscillator,

$$
\begin{aligned}
& \dot{x}_{1}=x_{2}, \\
& \dot{x}_{2}=-x_{1}-k_{0} x_{2} \Gamma\left(x_{1}, x_{2}\right),
\end{aligned}
$$

where $\Gamma\left(x_{1}, x_{2}\right)=x_{1}^{2}+x_{2}^{2}-\mu$, the constants $k_{0}$ and $\mu$ are positive, and for the sake of simplicity we have considered a unitary natural frequency. Then, adopting the feedback law

$$
x_{3}=\alpha_{0}\left(x_{1}, x_{2}\right)=-\frac{1}{g_{0}}\left[x_{1}+k_{0} x_{2} \Gamma\left(x_{1}, x_{2}\right)+f_{0}\right]
$$

system (2) is converted into system (3), and thus behaves as a stable nonlinear oscillator. Indeed, if we choose the Lyapunov function candidate

$$
V_{0}\left(x_{1}, x_{2}\right)=\frac{1}{4} \Gamma\left(x_{1}, x_{2}\right)^{2},
$$

and compute its orbital derivative for (3), it turns out that

$$
\dot{V}_{0}\left(x_{1}, x_{2}\right)=\left(x_{1} \dot{x}_{1}+x_{2} \dot{x}_{2}\right) \Gamma\left(x_{1}, x_{2}\right)=-k_{0} x_{2}^{2} \Gamma\left(x_{1}, x_{2}\right)^{2},
$$

so the function $V_{0}$ is monotone decreasing when both $x_{2} \neq 0$ and $\Gamma\left(x_{1}, x_{2}\right) \neq 0$, and it is constant when either $x_{2}=0$ or $\Gamma\left(x_{1}, x_{2}\right)=0$. Since the only equilibrium point of system (3) is the origin (which is unstable) and there are no other invariant subsets in the straight line $x_{2}=0$, from LaSalle's invariance principle, we conclude that the invariant manifold $x_{1}^{2}+x_{2}^{2}=\mu$, corresponding to $\Gamma\left(x_{1}, x_{2}\right)=0$, is globally attractive for all initial conditions different from the origin. The corresponding circle is then a stable (almost) globally attractive limit cycle.

Note that for $\Gamma\left(x_{1}, x_{2}\right) \neq 0$, system (3) can be written in the form

$$
\left[\begin{array}{l}
\dot{x}_{1} \\
\dot{x}_{2}
\end{array}\right]=\left[\begin{array}{cc}
0 & 1 \\
-1 & -k_{0} \Gamma
\end{array}\right]\left[\begin{array}{l}
x_{1} \\
x_{2}
\end{array}\right]=\left[\begin{array}{cc}
0 & \frac{1}{\Gamma} \\
-\frac{1}{\Gamma} & -k_{0}
\end{array}\right]\left[\begin{array}{l}
x_{1} \Gamma \\
x_{2} \Gamma
\end{array}\right]
$$

where $\left[x_{1} \Gamma, x_{2} \Gamma\right]^{T}=\partial V_{0} / \partial x$, so it has the structure of a generalized Hamiltonian system [1]. Also, if we took $k_{0}=0$ in (3), the system would become a harmonic oscillator and the origin would be a stable center, the origin being stable but not asymptotically stable. The geometric shape of $V_{0}$ is discussed in [7].

Remark 1: It is easy to see that this target system can also be obtained when the first equation of (1) takes the form:

$$
\dot{x}_{1}=\psi\left(x_{1}, x_{2}\right)
$$

where $\psi$ is invertible with respect to its second argument, so a function $\varphi$ exists such that $x_{2}=\varphi\left(x_{1}, \psi\right)$. Indeed, if we change the variables by the transformation $x_{2} \rightarrow z=\psi\left(x_{1}, x_{2}\right)$ the first two equations become

$$
\begin{aligned}
\dot{x}_{1} & =z \\
\dot{z} & =\bar{f}_{0}\left(x_{1}, z\right)+\bar{g}_{0}\left(x_{1}, z\right) x_{3}
\end{aligned}
$$

with

$$
\bar{f}_{0}\left(x_{1}, z\right)=\left.\left(\frac{\partial \psi}{x_{1}} z+\frac{\partial \psi}{x_{2}} f_{0}\right)\right|_{x_{2}=\varphi\left(x_{1}, z\right)} \text { and } \quad \bar{g}_{0}=\left.\left(\frac{\partial \psi}{x_{2}} g_{0}\right)\right|_{x_{2}=\varphi\left(x_{1}, z\right)} .
$$

Thus, the form (1) is recovered. However, for simplicity's sake, in the rest of this paper we will be concerned only with systems of form (1).

Remark 2: In this paper, we are interested in the generation of periodic movements in which the two first state variables behave as $\Gamma=x_{1}^{2}+x_{2}^{2}-\mu=0$. Obviously ,other choices of the target limit cycle $\hat{\Gamma}\left(x_{1}, x_{2}\right)=0$ are possible, and then the target system would be expressed by

$$
\left[\begin{array}{c}
\dot{x}_{1} \\
\dot{x}_{2}
\end{array}\right]=\left[\begin{array}{cc}
0 & \frac{1}{\widehat{\Gamma}} \\
-\frac{1}{\Gamma} & -k_{0}
\end{array}\right]\left[\begin{array}{l}
\widehat{\Gamma} \frac{\partial \widehat{\Gamma}}{\partial x_{1}} \\
\widehat{\Gamma} \frac{\partial \widehat{\Gamma}}{\partial x_{2}}
\end{array}\right]
$$

with Hamiltonian function $\widehat{H}=\widehat{\Gamma}^{2} / 2$. The study of the class of open-loop systems for which this more general target system is reachable by an appropriate control law is outside of the scope of this paper and will be considered elsewhere. 


\section{HIGHER-DIMENSION SYSTEMS}

In this section, the case $n>2$ is considered by using the backstepping method starting from the two-dimensional system (2). This two-dimensional system fulfills the following assumption, which is stated in a general way in order to apply the method recursively.

Assumption 1: For the system

$$
\dot{x}=F_{i}(x)+G_{i}(x) u,
$$

where $x \in \mathbb{R}^{i+2}$ is the state and $u \in \mathbb{R}$ is the control input, there is a continuously differentiable control law, $u=\alpha_{i}(x)$, and a smooth, non-negative, radially unbounded function, $V_{i}: \mathbb{R}^{i+2} \rightarrow \mathbb{R}$, such that ${ }^{1}$

$$
\left(\frac{\partial V_{i}}{\partial x}(x)\right)^{T}\left[F_{i}(x)+G_{i}(x) \alpha_{i}(x)\right] \leq 0, \quad \forall x \in \mathbb{R}^{i+2} .
$$

Below, $\mathcal{M}_{i}$ will denote the largest invariant set for which the equality of the previous expression holds. Under Assumption 1, LaSalle's invariance principle states that for system (7), with $u=\alpha_{i}(x)$, all the trajectories tend to the set $\mathcal{M}_{i}$.

In the first backstep, which corresponds to $i=0$, Assumption 1 is fulfilled by system (2) with $F_{0}=\left[x_{2}, f_{0}\right]^{T}, G_{0}=\left[0, g_{0}\right]^{T}$, $\alpha_{0}(x)$ given by (4) and $V_{0}=\Gamma^{2} / 4$. Also, from (6), it can be seen that

$$
\mathcal{M}_{0}=\{(0,0)\} \cup\left\{\left(x_{1}, x_{2}\right): \Gamma\left(x_{1}, x_{2}\right)=0\right\} .
$$

The following lemma is a slight modification of Lemma 2.8 in [6].

Lemma 1: Let system (7) be augmented in the following way:

$$
\begin{aligned}
\dot{x} & =F_{i}(x)+G_{i}(x) \xi \\
\dot{\xi} & =f_{i+1}(x, \xi)+g_{i+1}(x, \xi) u,
\end{aligned}
$$

with $g_{i+1} \neq 0$ and suppose that (9) satisfies Assumption 1 with $\xi \in \mathbb{R}$ as its control variable. Then, there is a feedback law that renders all the trajectories tending to the set $\mathcal{M}_{i+1}=\left\{(x, \xi): x \in \mathcal{M}_{i}, \xi=\alpha_{i}(x)\right\}$. One such control is

$$
\begin{aligned}
u \triangleq \alpha_{i+1}(x, \xi)= & \frac{1}{g_{i+1}}\left[-f_{i+1}-k_{i+1}\left[\xi-\alpha_{i}(x)\right]+\left(\frac{\partial \alpha_{i}}{\partial x}(x)\right)^{T}\left[F_{i}(x)+G_{i}(x) \xi\right]-\right. \\
& \left.\left(\frac{\partial V_{i}}{\partial x}(x)\right)^{T} G_{i}(x)\right], \quad k_{i+1}>0 .
\end{aligned}
$$

Proof: As is usual in the backstepping procedure, a new variable is introduced $z_{i+1} \triangleq \xi-\alpha_{i}(x)$ as the difference (error) between the augmented state variable and the desired virtual-control law. By using (11), the time derivative of $z_{k+1}$ is

$$
\dot{z}_{i+1}=-k_{i+1} z_{i+1}-G_{i}(x)^{T} \frac{\partial V_{i}}{\partial x}(x) .
$$

Defining the Lyapunov function candidate $V_{i+1}=V_{i}+z_{i+1}^{2} / 2$, it is easy to see that

$$
\dot{V}_{i+1}=\left(\frac{\partial V_{i}}{\partial x}(x)\right)^{T}\left[F_{i}(x)+G_{i}(x) \alpha_{i}(x)\right]-k_{i+1} z_{i+1}^{2} .
$$

Now, by applying LaSalle's invariance principle, the claim of the lemma is deduced.

Remark 3: Note that by renaming $(x, \xi)$ by $x$, and taking $F_{i+1}=\left[F_{i}+G_{i} \xi, f_{i+1}\right]^{T}, G_{i+1}=\left[0, g_{i+1}\right]^{T}, \alpha_{i+1}$ as in $(11)$ and $V_{i+1}=V_{i}+\left(\xi-\alpha_{i}\right)^{2} / 2$, Assumption 1 with $i$ replaced by $i+1$, also holds for the augmented system (9)-(10).

Below, it is shown that, after one application step of Lemma 1, the resulting closed-loop system preserves the structure of generalized Hamiltonian systems [1]. First, a slightly stronger assumption than the previous one is formulated.

Assumption 2: For the system

$$
\dot{x}=F_{i}(x)+G_{i}(x) u,
$$

where $x \in \mathbb{R}^{i+2}$ is the state and $u \in \mathbb{R}$ is the control input, there exists a continuously differentiable control law, $u=\alpha_{i}(x)$, and a smooth, non-negative, radially unbounded function, $V: \mathbb{R}^{i+2} \rightarrow \mathbb{R}$, such that

$$
F_{i}(x)+G_{i}(x) \alpha_{i}(x)=(J-R) \frac{\partial V_{i}}{\partial x}
$$

for some matrices $J=-J^{T}$ and $R=R^{T} \geq 0$. This means that the closed-loop system is a generalized Hamiltonian system.

${ }^{1}$ Throughout the paper all vectors, including the gradient $\nabla_{(\cdot)}=\frac{\partial}{\partial(\cdot)}$, are defined as column vectors. 
Lemma 2: Let the system (13) be augmented in the following way:

$$
\begin{aligned}
\dot{x} & =F_{i}(x)+G_{i}(x) \xi \\
\dot{\xi} & =f_{i+1}(x, \xi)+g_{i+1}(x, \xi) u,
\end{aligned}
$$

and suppose that (14) satisfies Assumption 2 with $\xi \in \mathbb{R}$ as its control. Then, Lemma 1 applies and, if the usual backstepping law (11) is used, the resulting closed-loop system is also a generalized Hamiltonian system.

Proof: From Assumption 2 it is easy to see that (8) holds and, thus, we can apply Lemma 1. Defining, as usual, the error variable $z_{i+1} \triangleq \xi-\alpha_{i}(x)$, and using variables $\left(x, z_{i+1}\right)$, and taking into account (12), the equations of the system are:

$$
\left[\begin{array}{c}
\dot{x} \\
\dot{z}_{i+1}
\end{array}\right]=\left[\begin{array}{cc}
J-R & G_{i}(x) \\
-G_{i}(x)^{T} & -k_{i+1}
\end{array}\right]\left[\begin{array}{c}
\frac{\partial V_{i}}{\partial x} \\
z_{i+1}
\end{array}\right],
$$

which means that the system is a generalized Hamiltonian system with Hamiltonian $V_{i}+z_{i+1}^{2} / 2$. As is well known, in passing to variables $(x, \xi)$ the generalized Hamiltonian character is preserved [8].

Remark 4: Notice that the applicability of this lemma goes beyond the stabilization of oscillations problem as stated here and, thus, it shows an interesting connection between backstepping and generalized Hamiltonian systems.

Remark 5: By applying the above lemmas in recursive form and starting with the target system defined in the previous section, the control law for systems of form (1) of arbitrary dimension $n$ can be obtained so that the resulting closed-loop system is an oscillating generalized Hamiltonian system, which presents an almost-globally stable limit cycle. The limit cycle corresponds to the set

$$
L C=\left\{\left(x_{1}, x_{2}, z_{1}, \ldots, z_{m}\right) \in \mathbb{R}^{n}: \Gamma\left(x_{1}, x_{2}\right)=0, z_{i}=0, i=1, \ldots, m\right\}
$$

For a general $n$, the successive virtual control laws $\alpha_{i}, i=1, \ldots, m$ can be obtained and, thus, variables $z_{i}=x_{i+2}-\alpha_{i-1}, i=$ $1, \ldots, m$ can be defined. It is easy to see that working in $\left(x_{1}, x_{2}, z_{1}, z_{2}, \ldots, z_{m}\right)$ variables the following expression for the closed-loop system is obtained:

$$
\left[\begin{array}{c}
\dot{x}_{1} \\
\dot{x}_{2} \\
\dot{z}_{1} \\
\dot{z}_{2} \\
\vdots \\
\dot{z}_{m-1} \\
\dot{z}_{m}
\end{array}\right]=\left[\begin{array}{ccccccc}
0 & \frac{1}{\Gamma} & 0 & 0 & \cdots & 0 & 0 \\
-\frac{1}{\Gamma} & -k_{0} & g_{0} & 0 & \cdots & 0 & 0 \\
0 & -g_{0} & -k_{1} & g_{1} & \cdots & 0 & 0 \\
0 & 0 & -g_{1} & -k_{2} & \cdots & 0 & 0 \\
\vdots & \vdots & \vdots & \vdots & \ddots & \vdots & \vdots \\
0 & 0 & 0 & 0 & \cdots & -k_{m-1} & g_{m-1} \\
0 & 0 & 0 & 0 & \cdots & -g_{m-1} & -k_{m}
\end{array}\right]\left[\begin{array}{c}
x_{1} \Gamma \\
x_{2} \Gamma \\
z_{1} \\
z_{2} \\
\vdots \\
z_{m-1} \\
z_{m}
\end{array}\right]
$$

where, $\Gamma\left(x_{1}, x_{2}\right) \neq 0$ and, for brevity, the arguments of functions $g_{i}$ have been omitted. Actually, they should be $g_{i}\left(x_{1}, x_{2}, z_{1}+\right.$ $\left.\alpha_{0}, \ldots, z_{i}+\alpha_{i-1}\right)$. Thus, the closed-loop system is again a generalized Hamiltonian one. As was pointed out above, the generalized Hamiltonian structure is preserved when the transformation $\left(x_{1}, x_{2}, z_{1}, z_{2}, \ldots, z_{m}\right) \rightarrow\left(x_{1}, x_{2}, x_{3}, x_{4}, \ldots, x_{n}\right)$ is performed.

Remark 6: Upon applying LaSalle's invariance principle to system (16), it is easy to see that, in order to achieve the objective, the requirement $k_{i}>0, i=0, \ldots, m$ can be relaxed to $k_{i} \geq 0, i=0, \ldots, m-1, k_{m}>0$.

Since Assumptions 1 and 2 are always fulfilled by the first two equations of (1), and in successive steps, as shown above, the previous results can be stated by means of the following theorem.

Theorem 1: Given a strict-feedback system of form (1) where functions $g_{i}$ do not vanish, a nonlinear feedback law exists that renders this system a robust oscillator, in the sense that the only limit set is one almost globally attractive limit cycle.

Remark 7: Notice that class (1) includes linear systems in Brunovsky canonical form [9] and, thus, the method presented is applicable, among others, to feedback linearizable systems. Notice also that although the open-loop system is, in this case, linear, the closed-loop system is nonlinear as it has to be in order to present robust oscillations.

Example 1: Consider the following linear, three-dimensional system in Brunovsky form

$$
\begin{aligned}
& \dot{x}_{1}=x_{2}, \\
& \dot{x}_{2}=x_{3}, \\
& \dot{x}_{3}=u .
\end{aligned}
$$

Starting with the system obtained in Section II, only one backstep is needed with $i=0, \xi=x_{3}, F_{0}\left(x_{1}, x_{2}\right)=\left[x_{2}, 0\right]^{T}$, $G_{0}\left(x_{1}, x_{2}\right)=[0,1]^{T}, \alpha_{0}(x)$ given by (4) and $V=V_{0}$. The application of Eq. (11) yields:

$$
u=-x_{2}-\left(x_{1}+x_{3}\right)\left(k_{1}+2 k_{0} x_{2}^{2}\right)-\left[\left(1+k_{0} k_{1}\right) x_{2}+k_{0} x_{3}\right] \Gamma\left(x_{1}, x_{2}\right),
$$


with $k_{0} \geq 0$ and $k_{1}>0$, which is a nonlinear feedback law in spite of the fact that the open-loop system is linear. As a result of this control law, for every initial condition except the origin, the trajectories of the closed-loop system tend to the limit cycle $\left\{\Gamma\left(x_{1}, x_{2}\right)=0, x_{1}=-x_{3}\right\}$.

Furthermore, following Lemma 2 the resulting generalized Hamiltonian system in $\left(x_{1}, x_{2}, z\right)$ coordinates is:

$$
\left[\begin{array}{c}
\dot{x}_{1} \\
\dot{x}_{2} \\
\dot{z}
\end{array}\right]=\left[\begin{array}{ccc}
0 & \frac{1}{\Gamma} & 0 \\
-\frac{1}{\Gamma} & -k_{0} & 1 \\
0 & -1 & -k_{1}
\end{array}\right]\left[\begin{array}{l}
\frac{\partial V_{1}}{\partial x_{1}} \\
\frac{\partial V_{1}}{\partial x_{2}} \\
\frac{\partial V_{1}}{\partial z}
\end{array}\right]
$$

with $z=x_{3}+x_{1}+k_{0} x_{2} \Gamma\left(x_{1}, x_{2}\right)$ and $V_{1}\left(x_{1}, x_{2}, x_{3}\right)=V_{0}\left(x_{1}, x_{2}\right)+z^{2} / 2$. Returning to the $\left(x_{1}, x_{2}, x_{3}\right)$ variables, it is found $\dot{x}=[J-R] \partial \bar{V}_{1} / \partial x$, with $\bar{V}_{1}\left(x_{1}, x_{2}, x_{3}\right)=\Gamma^{2} / 4+\left(x_{1}+x_{3}+k_{0} x_{2} \Gamma\right)^{2} / 2$,

$$
J=\frac{1}{\Gamma}\left[\begin{array}{ccc}
0 & 1 & -k_{0}\left(\Gamma+2 x_{2}^{2}\right) \\
-1 & 0 & 1+2 k_{0} x_{1} x_{2}+\Gamma \\
k_{0}\left(\Gamma+2 x_{2}^{2}\right) & -1-2 k_{0} x_{1} x_{2} \Gamma & 0
\end{array}\right]
$$

and

$$
R=\left[\begin{array}{ccc}
0 & 0 & 0 \\
0 & k_{0} & -k_{0}^{2}\left(\Gamma+2 x_{2}^{2}\right) \\
0 & -k_{0}^{2}\left(\Gamma+2 x_{2}^{2}\right) & k_{0}^{3}\left(\Gamma+2 x_{2}^{2}\right)^{2}+k_{1}
\end{array}\right]
$$

Thus, $J$ and $R$ display the hidden generalized Hamiltonian structure of system (17)-(18).

Example 2: Let us illustrate the applicability of the method with a physical example, a magnetic levitation ball system. The system, which consists of an iron ball in a vertical magnetic field created by a single electromagnet, is depicted in Fig. 1. The position of the ball is denoted by $x_{1}$, with the $x_{1}$-axis oriented upwards. The magnetic flux in the inductance is denoted by $x_{3}$.

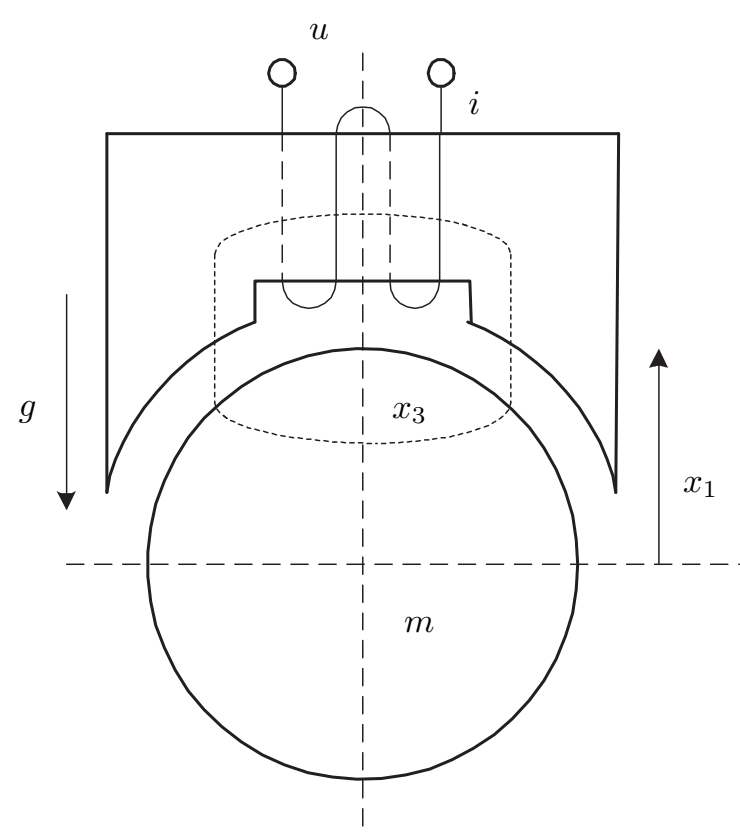

Fig. 1. Magnetic levitation system.

The open-loop equations of the magnetic levitation system [10] are given by

$$
\begin{aligned}
& \dot{x}_{1}=x_{2} \\
& \dot{x}_{2}=\frac{1}{2 m} x_{3}^{2}-g \\
& \dot{x}_{3}=-R\left(1-x_{1}\right) x_{3}+u
\end{aligned}
$$

where $x_{2}=\dot{x}_{1}, m$ is the mass of the ball, $R$ is the electrical resistance of the electromagnet and $g$ is the gravity acceleration. 
In order to write the model in the form (1), the change of variables $w=x_{3}^{2} / 2$ is introduced. Notice that it is reasonable to only consider positive values for $x_{3}$ since the magnetic force has to compensate for gravity. In this way, the model becomes

$$
\begin{aligned}
\dot{x}_{1} & =x_{2} \\
\dot{x}_{2} & =\frac{w}{m}-g \\
\dot{w} & =-2 R\left(1-x_{1}\right) w+u \sqrt{2 w} .
\end{aligned}
$$

Thus, $f_{0}=-g, g_{0}=1 / m, f_{1}=-2 R\left(1-x_{1}\right) w$ and $g_{1}=\sqrt{2 w}$. The expression for $\alpha_{0}$ can be obtained from (4)

$$
\alpha_{0}=m\left(g-x_{1}-k_{0} x_{2} \Gamma\right) .
$$

Using (11) the control law is obtained

$$
\begin{aligned}
u= & \alpha_{1}=\frac{1}{\sqrt{2 w}}\left(2 R\left(1-x_{1}\right) w-k_{1}\left(w-\alpha_{0}\right)-\right. \\
& \left.m\left(1+2 k_{0} x_{1} x_{2}\right) x_{2}-m k_{0}\left(\Gamma+2 x_{2}^{2}\right)\left(\frac{w}{m}-g\right)-\frac{\Gamma x_{2}}{m}\right) .
\end{aligned}
$$

The final control law is obtained by undoing the change of variables

$$
\begin{aligned}
u= & \alpha_{1}=\frac{1}{x_{3}}\left(2 R\left(1-x_{1}\right) \frac{x_{3}^{2}}{2}-k_{1}\left(\frac{x_{3}^{2}}{2}-m\left(g-x_{1}-k_{0} x_{2} \Gamma\right)\right)-\right. \\
& \left.m\left(1+2 k_{0} x_{1} x_{2}\right) x_{2}-m k_{0}\left(\Gamma+2 x_{2}^{2}\right)\left(\frac{x_{3}^{2}}{2 m}-g\right)-\frac{\Gamma x_{2}}{m}\right) .
\end{aligned}
$$

This feedback law is valid provided that $x_{3}>0$, which limits the domain of attraction of the limit cycle. It has been checked by simulation and yields the desired behavior. The results for $m=0.5, g=9.8, R=2, k_{0}=0.5, k_{1}=1, \mu=1$ and initial conditions $\left(x_{1}, x_{2}, x_{3}\right)=(0.1,0,1)$ are depicted in Fig. 2. The top-left graph shows the projection of the trajectory on the $x_{1}-x_{2}$ plane. It can be seen that the closed curve $\Gamma=0$ is reached. The top-right graph shows the time evolution of the state variables, while the bottom-left graph shows the control signal behaviour. Finally, in the bottom-right graph the evolution of the Lyapunov function $V_{1}=\frac{\Gamma^{2}}{4}+\frac{\left(w-\alpha_{0}\right)^{2}}{2}$ is represented.
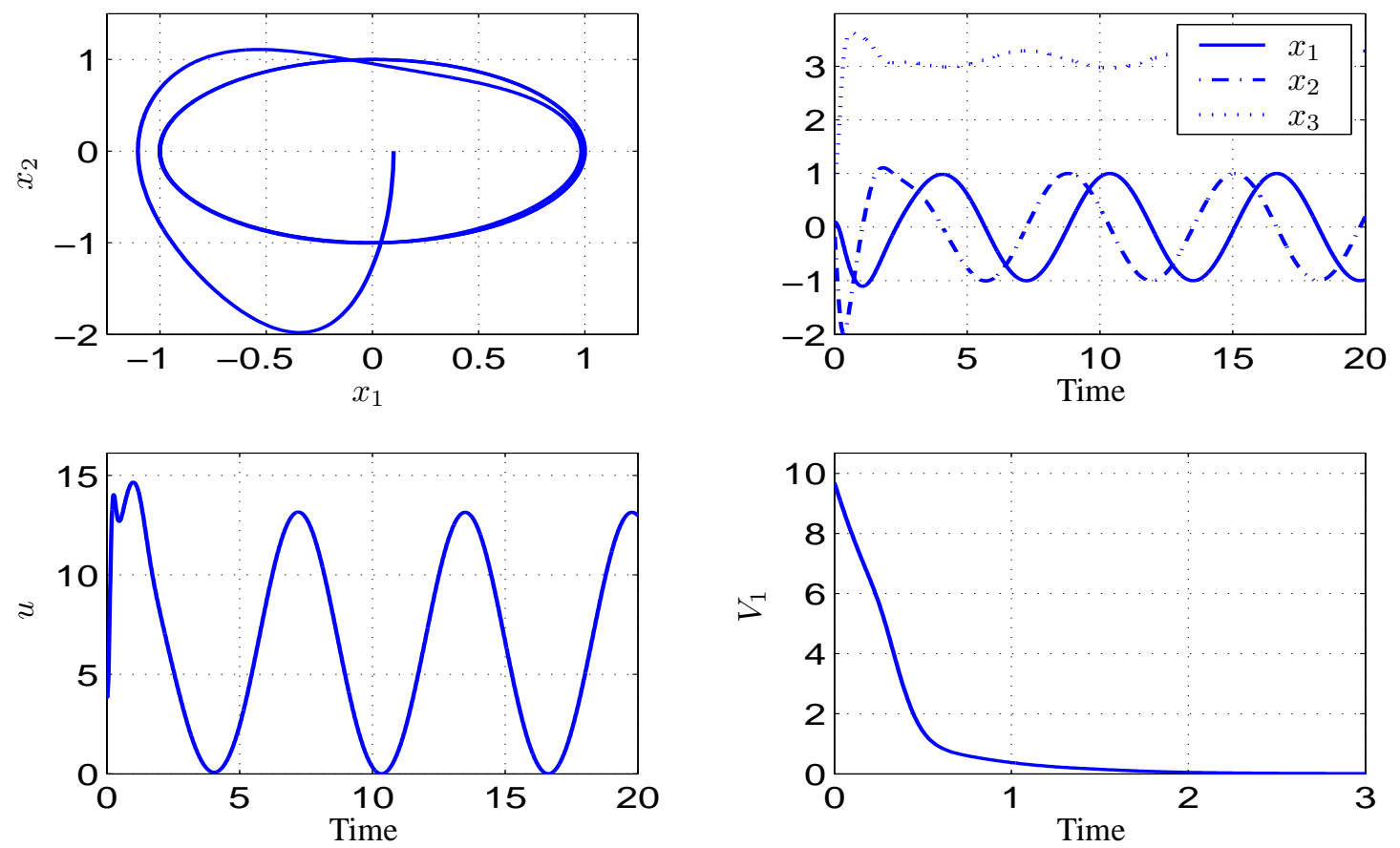

Fig. 2. Simulation results for the magnetic levitation system.

The following theorem states that the limit cycle, which acts as the only attractor of the controlled system, can also be conceived of as a consequence of a dynamic bifurcation [11] after varying the value of parameter $\mu$.

Theorem 2: System (1) with the control law resulting from the iterative application of (11) undergoes a supercritical Hopf bifurcation at $\mu=0$. 
Proof: See the Appendix.

The fact that a Hopf bifurcation occurs is interesting because it gives as a by-product a point stabilization law on the "other side" of the bifurcation point. In other words, by changing the bifurcation parameter $\mu$ from positive to negative values we have a method by which to go in a continuous way from a controller that makes the system oscillatory to an equilibrium stabilizing controller, in the sense of asymptotic stabilization of an equilibrium point.

Remark 8: It is well known that the Hopf bifurcation takes place in a space of dimension two. For higher order systems there is a reduction procedure based on the center manifold theorem [12]. This theorem gives a procedure for reducing an $n$-dimensional system to a two-dimensional one where the Hopf bifurcation takes place. This procedure is known as the Reduction Principle. Here we proceed in the opposite sense. We built an appropriate feedback law by considering first a two-dimensional subsystem of the whole system that undergoes a Hopf bifurcation. Then, by backstepping, the full system is rendered oscillatory. Therefore, the method proposed here gives rise to a kind of extension method where the Hopf bifurcation is extended to a higher-order system.

\section{CONCLUSIONS}

In this paper, we have presented a technique for obtaining stable and robust oscillations in a class of single-input nonlinear cascade systems. To accomplish this, a feedback law was introduced that converts an appropriate second-order subsystem into a damped harmonic oscillator. Then, by backstepping, a feedback law is recursively obtained for the full system. It is shown that, as a result of applying backstepping, the oscillator evolves into a stable limit cycle, and therefore the oscillations are robust. It is also shown that the resulting closed-loop system displays the generalized Hamiltonian structure. The birth of the limit cycle responsible for the oscillations has been associated with the occurrence of a Hopf bifurcation.

\section{ApPendix: Checking THE Hopf BIfURCATION CONDitions AT $\mu=0$}

We start from the linearization matrix $A(\mu)$ of system (16) at the origin, which yields

$$
A(\mu)=\left[\begin{array}{ccccccc}
0 & 1 & 0 & 0 & \cdots & 0 & 0 \\
-1 & \mu k_{0} & g_{0} & 0 & \cdots & 0 & 0 \\
0 & \mu g_{0} & -k_{1} & g_{1} & \cdots & 0 & 0 \\
0 & 0 & -g_{1} & -k_{2} & \cdots & 0 & 0 \\
\vdots & \vdots & \vdots & \vdots & \ddots & \vdots & \vdots \\
0 & 0 & 0 & 0 & \cdots & -k_{m-1} & g_{m-1} \\
0 & 0 & 0 & 0 & \cdots & -g_{m-1} & -k_{m}
\end{array}\right]
$$

where functions $g_{i}$ are evaluated at $\left(x_{1}=0, x_{2}=0, z_{1}=0, \ldots, z_{m}=0\right)$.

It will be shown that this matrix has an eigenvalue pair $\sigma(\mu) \pm j \omega(\mu)$ that crosses the imaginary axis at $\mu=0$ with a positive velocity (that is, $\sigma^{\prime}(0)>0$, the so-called transversality condition), while the other eigenvalues have negative real parts. All these are necessary conditions for the occurrence of a supercritical Hopf bifurcation at $\mu=0$ that gives rise to a stable limit cycle for $\mu>0$.

Let us define for $i=1,2, \ldots, m$ the determinants

$$
\Delta_{i}(s)=\operatorname{det}\left[\begin{array}{ccccc}
s+k_{i} & -g_{i} & \cdots & 0 & 0 \\
g_{i} & s+k_{i+1} & \cdots & 0 & 0 \\
\vdots & \vdots & \ddots & \vdots & \vdots \\
0 & 0 & \cdots & s+k_{m-1} & -g_{m-1} \\
0 & 0 & \cdots & g_{m-1} & s+k_{m}
\end{array}\right]
$$

so that $\Delta_{m}(s)=s+k_{m}$, and they can be recursively computed by taking $\Delta_{m+1}(s) \equiv 1$ and noting that

$$
\Delta_{i}(s)=\left(s+k_{i}\right) \Delta_{i+1}(s)+g_{i}^{2} \Delta_{i+2}(s)
$$

for $i=1,2, \ldots, m-1$. The following result will be useful.

Lemma 3: Assume that all the constants $k_{1}, k_{2}, \ldots, k_{m}$ are non-negative and the last one is positive. If $s=\sigma+j \omega \in \mathbb{C}$ has a non-negative real part (that is, $\sigma \geq 0$ ), then $\Delta_{i}(s) \neq 0$ and the quotient $\Delta_{i}(s) / \Delta_{i+1}(s)$ has a positive real part for $i=1,2, \ldots, m$.

Proof: The statement is trivial when $i=m$. From (25), we can write

$$
\frac{\Delta_{m-1}(s)}{\Delta_{m}(s)}=s+k_{m-1}+g_{m-1}^{2}\left(\frac{\Delta_{m}(s)}{\Delta_{m+1}(s)}\right)^{-1},
$$

so that the left hand side has a positive real part and, thus $\Delta_{m-1}(s) \neq 0$. Clearly, we can proceed backwards up to $i=1$. 
If we now compute the characteristic polynomial of $A(\mu)$, we get

$$
p(s, \mu)=\operatorname{det}[s I-A(\mu)]=\left(s^{2}-\mu k_{0} s+1\right) \Delta_{1}(s)-\mu g_{0}^{2} s \Delta_{2}(s),
$$

and then $p(s, 0)=\left(s^{2}+1\right) \Delta_{1}(s)$. From Lemma 3, all the roots of the polynomial $\Delta_{1}$ have negative real parts (i.e. it is a Hurwitz polynomial), and we conclude that for small $\mu$ matrix $A(\mu)$ has $n-2$ eigenvalues with negative real parts and a single eigenvalue pair $s_{ \pm}(\mu)=\sigma(\mu) \pm j \omega(\mu)$ with $\sigma(0)=0, \omega(0)=1$. Below, it is shown that $\sigma^{\prime}(0)>0$.

Applying the Implicit Function Theorem to the equation $p(s, \mu)=0$ in a neighborhood of $\left(s_{+}(\mu), \mu\right)=(j, 0)$ yields

$$
\frac{\partial p}{\partial s}\left(s_{+}(0), 0\right) \frac{d s_{+}}{d \mu}(0)+\frac{\partial p}{\partial \mu}\left(s_{+}(0), 0\right)=0
$$

and using (26), we obtain the equation

$$
2 j \Delta_{1}(j) s_{+}^{\prime}(0)-k_{0} j \Delta_{1}(j)-j g_{0}^{2} \Delta_{2}(j)=0
$$

SO

$$
s_{+}^{\prime}(0)=\frac{1}{2}\left(k_{0}+g_{0}^{2} \frac{\Delta_{2}(j)}{\Delta_{1}(j)}\right) .
$$

Thus, again from Lemma 3, we see that $\sigma^{\prime}(0)=\Re\left[s_{+}^{\prime}(0)\right]$ is positive and the transversality condition for the occurrence of a Hopf bifurcation is satisfied.

\section{ACKNOWLEDGMENT}

The authors greatly appreciate the suggestions by Emilio Freire, Fabio Gómez-Estern and by the anonymous referees of this paper. This work has been supported under MCyT-FEDER grants DPI2003-00429 and DPI2001-2424-C02-01.

\section{REFERENCES}

[1] A. van der Schaft, $L_{2}$-Gain and Passivity Techniques in Nonlinear Control. Springer-Verlag, 1989.

[2] R. Goldwyn and K. J. Cox, "Limit cycle construction using Liapunov functions," IEEE Trans. Automat. Control, vol. 10, No. 1, pp. 97-99, 1965.

[3] D. Green, "Synthesis of systems with periodic solutions satisfying $\mathbf{N}(\mathbf{x})=\mathbf{0}$," IEEE Trans. Circuits and Systems, vol. 31, No. 4, pp. 317-326, 1984.

[4] K. Hirai and K. Maeda, "A method of limit-cycle synthesis and its applications," IEEE Trans. on Circuit Theory, pp. 631-633, 1972.

[5] J. Aracil, F. Gómez-Estern, and F. Gordillo., "A family of oscillating generalized Hamiltonian systems," in 2nd IFAC Workshop on Lagrangian and Hamiltonian Methods for Nonlinear Control, Seville (Spain), 2003, pp. 93-98.

[6] M. Krstic, I. Kanellakopoulos, and P. Kokotovic, Nonlinear and Adaptive Control Design. Wiley, 1995.

[7] J. Aracil, F. Gordillo, and J. Acosta, "Stabilization of oscillations in the inverted pendulum," in XV IFAC World Congress, 2002.

[8] K. Fujimoto and T. Sugie, "Canonical transformation and stabilization of generalized Hamiltonian systems," Systems \& Control Letters, vol. 42, no. 3, pp. 217-227, March 2000.

[9] R. Marino and P. Tomei, Nonlinear Control Design. Prentice Hall, 1995.

[10] O. R, A. Loria, P. Niklasson, and H. Sira-Ramrez, Passivity-based Control of Euler-Lagrange Systems. Springer-Verlag, 1998.

[11] J. Marsden and M. McCraken, The Hopf Bifurcation and Its Applications. New York: Springer-Verlag, 1976.

[12] J. Carr, Applications of Centre Manifold Theory. New York: Springer-Verlag, 1981. 